\title{
Time delay in atomic photoionization with circularly polarized light
}

\author{
I. A. Ivanov ${ }^{*}$ and A. S. Kheifets ${ }^{\dagger}$ \\ Research School of Physical Sciences, Australian National University, Canberra, Australian Capital Territory 0200, Australia
}

(Received 16 December 2012; published 7 March 2013)

\begin{abstract}
We study time delay in atomic photoionization by circularly polarized light. By considering the Li atom in an excited $2 p$ state, we demonstrate a strong time-delay asymmetry between the photoemission of the target electrons that are co- and counter-rotating with the electromagnetic field in the polarization plane. In addition, we observe the time-delay sensitivity to the polar angle of the photoelectron emission in the polarization plane. This modulation depends on the shape and duration of the electromagnetic pulse.
\end{abstract}

DOI: 10.1103/PhysRevA.87.033407

PACS number(s): $32.80 . \mathrm{Rm}, 32.80 . \mathrm{Fb}, 42.50 . \mathrm{Hz}$

\section{INTRODUCTION}

Atomic or molecular photoionization in a circularly polarized electromagnetic (EM) field exhibits a number of features that are absent in the case of linear polarization. One such effect is dependence of the photoionization probability on the direction of the rotation of the target electron relative to the polarization plane of light. Let us assume that the EM field is right circularly polarized in the $(x, y)$ plane. We call the target electron co- or counter-rotating with the field if its angular momentum projection on the $z$ axis is $m>0$ or $m<0$, respectively. This difference of ionization probabilities for co- or counter-rotating electrons is present both in the multiphoton and tunneling regimes. However, it manifests itself quite differently. In the multiphoton regime, the ionization probability is larger for the corotating electrons $[1,2]$. In contrast, in the tunneling regime, ionization for the counter-rotating electron dominates [3]. The latter effect may have important implications for the angular attosecond streaking technique, which makes it possible to trace electronic motion in atoms and molecules with the resolution of several attoseconds [4].

The earlier works [1-3] focused on the asymmetry in photoionization cross sections for the co- and counter-rotating target electrons. In the present study, we supplement these works with time-delay analysis. The notion of time delay was initially introduced by Wigner [5] for scattering phenomena and applied subsequently to photoionization [6]. A number of benchmark experimental and theoretical results have been obtained in studies of atomic photoionization using this approach [4,7-9]. These studies have been extended to molecules [10] and double-electron photoionization [11]. In the present work, we calculate the time delay following atomic photoionization by a circularly polarized EM field. For a numerical illustration, we consider ionization of the lithium atom prepared in the excited $2 p$ state. Such a state is readily experimentally accessible (see, e.g., [12]). Our particular interest will be in the time-delay asymmetry between the coand counter-rotating target electrons with $m= \pm 1$.

The paper is organized as follows. In Sec. II we outline our theoretical model. In Sec. III we present our numerical results for the photoelectron spectrum, momentum distribution, and

\footnotetext{
*Igor.Ivanov@anu.edu.au

†A.Kheifets@anu.edu.au
}

time delay. We conclude in Sec. IV by outlining the key features of the observed effects. Atomic units are used throughout the paper unless otherwise specified.

\section{THEORETICAL MODEL}

We solve the time-dependent Schrödinger equation (TDSE) for an atom with a single active electron,

$$
i \frac{\partial \Psi(\boldsymbol{r})}{\partial t}=\left[\hat{H}_{\mathrm{atom}}+\hat{H}_{\mathrm{int}}(t)\right] \Psi(\boldsymbol{r}) .
$$

Here $\hat{H}_{\text {atom }}$ is the Hamiltonian of the field-free atom with an effective one-electron potential [13]. $\hat{H}_{\text {int }}(t)$ describes the interaction with the EM field, either in the length or velocity gauges:

$$
\hat{H}_{\text {int }}(t)=\left\{\begin{array}{l}
\boldsymbol{E}(t) \cdot \hat{\boldsymbol{r}} \\
\boldsymbol{A}(t) \cdot \hat{\boldsymbol{p}}, \quad \text { where } \boldsymbol{A}(t)=-\int_{-T_{1} / 2}^{t} \boldsymbol{E}(\tau) d \tau
\end{array}\right.
$$

The field is right-circularly polarized, propagating along the $z$ direction (which is assumed to be the quantization axis):

$$
E_{x}=\mathcal{E} f(t) \cos \omega t, \quad E_{y}=\mathcal{E} f(t) \sin \omega t .
$$

Here $f(t)=\cos ^{2}\left(\pi t / T_{1}\right)$ is the pulse envelope. The field is present on the interval $\left(-T_{1} / 2, T_{1} / 2\right)$. We measure the pulse duration in units of the optical cycle $T_{1} / T$ where $T=2 \pi / \omega$.

The solution of Eq. (1) is sought in the form of a partial wave expansion

$$
\Psi(\boldsymbol{r}, t)=\sum_{l=0}^{L_{\max }} \sum_{\mu=-l}^{l} f_{l \mu}(r, t) Y_{l \mu}(\theta, \phi) .
$$

The radial part of the TDSE is discretized on the grid with the stepsize $\delta r=0.05$ a.u. in a box of the size $R_{\max }$. The convergence of Eq. (4) with $L_{\max }$ and $R_{\max }$ depends on the field strength $\mathcal{E}$. This convergence is tested thoroughly in each numerical example presented below. To propagate the wave function (4) in time, we use the matrix iteration method developed in [14] and further tested in strong-field ionization calculations $[15,16]$.

We project the solution of the TDSE at the end of the laser pulse at $t=T_{1} / 2$ on the set of the incoming scattering states:

$$
\psi_{\boldsymbol{k}}^{(-)}(\boldsymbol{r})=\sum_{l \mu} i^{l} e^{-i \delta_{l}} Y_{l \mu}^{*}\left(\boldsymbol{n}_{\boldsymbol{k}}\right) Y_{l \mu}\left(\boldsymbol{n}_{\boldsymbol{r}}\right) R_{k l}(r),
$$

where $\boldsymbol{n}_{\boldsymbol{k}}=\boldsymbol{k} / k$, and $\boldsymbol{n}_{\boldsymbol{r}}=\boldsymbol{r} / \boldsymbol{r}$ are unit vectors in the direction of $\boldsymbol{k}$ and $\boldsymbol{r}$, respectively. This projection gives us a set of 
coefficients

$$
a_{\boldsymbol{k}}=\left\langle\psi_{\boldsymbol{k}}^{(-)} \mid \Psi(t)\right\rangle e^{i E_{k} t} .
$$

The squared moduli of these coefficients determine the photoelectron spectrum. The energy derivative of the phase of the coefficients (6) gives the photoelectron group delay, which is also known as the Wigner time delay. An equivalent form, which is more convenient in practical calculations, is the following (more details about derivation of this equation can be found in the Appendix):

$$
\tau_{0}=\operatorname{Im}\left(\frac{\boldsymbol{n}_{\boldsymbol{q}}}{q} \frac{\partial a_{\boldsymbol{k}}}{\partial \boldsymbol{k}}\right) .
$$

Here the derivative is computed at the point $\boldsymbol{k}=\boldsymbol{q}$, corresponding to the asymptotic momentum of the photoelectron in the field-free zone [16-18], and $\boldsymbol{n}_{\boldsymbol{q}}=\boldsymbol{q} / \boldsymbol{q}$.

We notice that when a single partial wave $l$ in the sum (5) is dominant, the photoelectron time delay is simply the energy derivative of the corresponding elastic scattering phase shift $\tau_{0}=d \delta_{l} / d E=k^{-1} d \delta_{l} / d k \times 24$ as. Here one atomic unit of time is equated to 24 as.

The time delay $\tau_{0}$ has a transparent physical meaning $[5,6]$. It appears as a coefficient in the asymptotic expression for the trajectory of the crest of the wave packet

$$
\boldsymbol{r}(t) \sim \boldsymbol{q}\left(t-\tau_{0}\right)+\boldsymbol{r}^{\prime}(t) .
$$

Here the term $\boldsymbol{r}^{\prime}(t)$ gives well-known Coulomb corrections to the trajectory which grows logarithmically with $t$ for large $t$.

\section{NUMERICAL RESULTS}

For numerical illustrations, we perform photoionization calculations of the lithium atom in the $2 p$ excited state. We use circular polarized field pulses with the carrier frequency $\omega=0.5$ a.u. (13.6 eV) and the pulse durations $T_{1}$ varying from 3 to 10 optical cycles ( 1 to $3 \mathrm{fs}$ ). The field strength ranges from $\mathcal{E}=0.01$ to 0.1 a.u., which corresponds to a field intensity of $3.5 \times 10^{12}$ to $3.5 \times 10^{14} \mathrm{~W} / \mathrm{cm}^{2}$.

\section{A. Photoelectron spectrum}

We start our calculations with the photoelectron spectrum integrated over all the escape directions. We test convergence of our results with respect to the values of $L_{\max }$ in Eq. (4) and the box size $R_{\max }$ as well as the gauge invariance between the length and velocity forms of the atom-EM field interaction (2). We illustrate this test in Fig. 1 using the most challenging condition of the strong field ionization with $\mathcal{E}=0.1$ a.u. The pulse duration is 10 optical cycles. In this figure we show the photoelectron spectrum following ionization of the $2 p_{+1}$ initial state. The velocity gauge results with the pair of values $L_{\max } / R_{\max }$ (a.u.) $=12 / 500,10 / 500$, and $10 / 900$ as well as the length gauge result with $L_{\max } / R_{\max }(\mathrm{au})=12 / 500$ are indistinguishable on the scale of the figure. A small peak at higher electron energy of $\simeq 23 \mathrm{eV}$ is a manifestation of a two-photon absorption. Such two-photon processes, as can be seen from Fig. 1, are not very important as long as we are interested in the electron spectra only. As we see below, the situation is rather different if we are interested in computing the time delay.

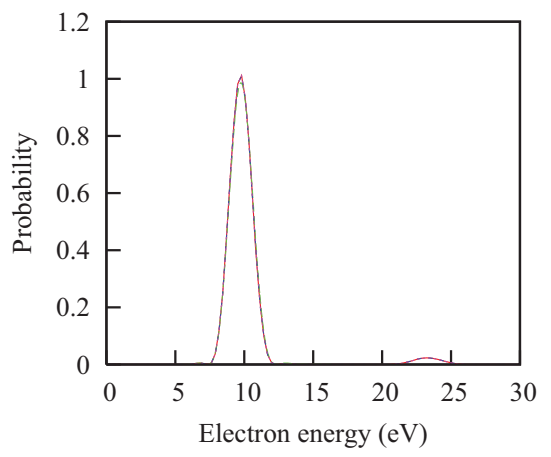

FIG. 1. (Color online) The photoelectron spectrum of Li ionization from the $2 p_{+1}$ state. The field parameters are $\omega=0.5$ au and $\mathcal{E}=0.1$ au and the pulse duration is 10 optical cycles. The calculations with the following pairs of values $L_{\max } / R_{\max }$ (a.u.) are plotted. Solid line (red online), 12/500; short dashed line (blue online), 10/500; dots (magenta online), 10/900, all in the velocity gauge; dashed line (green online), 12/500, the length gauge.

\section{B. Photoelectron momentum distribution}

In this and the following section, we confine our study to the equatorial $(x, y)$ plane in which most of the photoelectrons escape. In Fig. 2 we present the photoelectron momentum distributions in this plane for various field strengths and pulse durations. The upper row of Fig. 2 shows the photoelectron momentum distribution obtained for the weak field of $\mathcal{E}=0.01$ a.u. and the pulse length of 3 optical cycles. This weak field regime can be analyzed within the lowest order perturbation theory (LOPT). Under this condition, an electron in the $2 p_{+1}$ initial state, upon absorption of a photon from the circularly polarized EM field, Eq. (3), ends up in the $d$ partial wave with the angular momentum projection $\mu=2$. An electron ionized from the $2 p_{-1}$ state ends up in the superposition of the continuum $s$ and $d$ states with $\mu=0$. In both cases, the photoelectron angular distribution in the equatorial plane will be independent on the polar angle $\phi$, which is clearly seen in Fig. 2.

Results for the higher field strengths are shown in the second row ( $\mathcal{E}=0.05$ a.u.) and third and fourth rows ( $\mathcal{E}=0.1$ a.u.). Unlike in the weak field regime, the photoelectron angular distributions for a short pulse of 3 optical cycles show some angular structure, which is due to the higher order processes.

\section{Photoelectron time delay}

In Fig. 3 we present the time-delay results as a function of the polar angle for the photoelectrons escaping in the equatorial plane. Also shown are the energy derivatives of the scattering phase shifts in the $s$ and $d$ partial waves, computed at the energies corresponding to the peak of the photoelectron wave packet in the momentum space. The three sets of panels display the time-delay results for the field strengths of $\mathcal{E}=0.01$ a.u. (top), $\mathcal{E}=0.05$ a.u. (middle), and $\mathcal{E}=0.1$ a.u. (bottom). The pulse duration is $T_{1} / T=3$ and 10 for the left and right sets of panels, respectively.

Let us first discuss the weak field regime of $\mathcal{E}=0.01$ a.u. for which the perturbative treatment is applicable. Unlike the photoelectron angular distributions displayed in Fig. 2, the time-delay results for the short pulse duration show a 

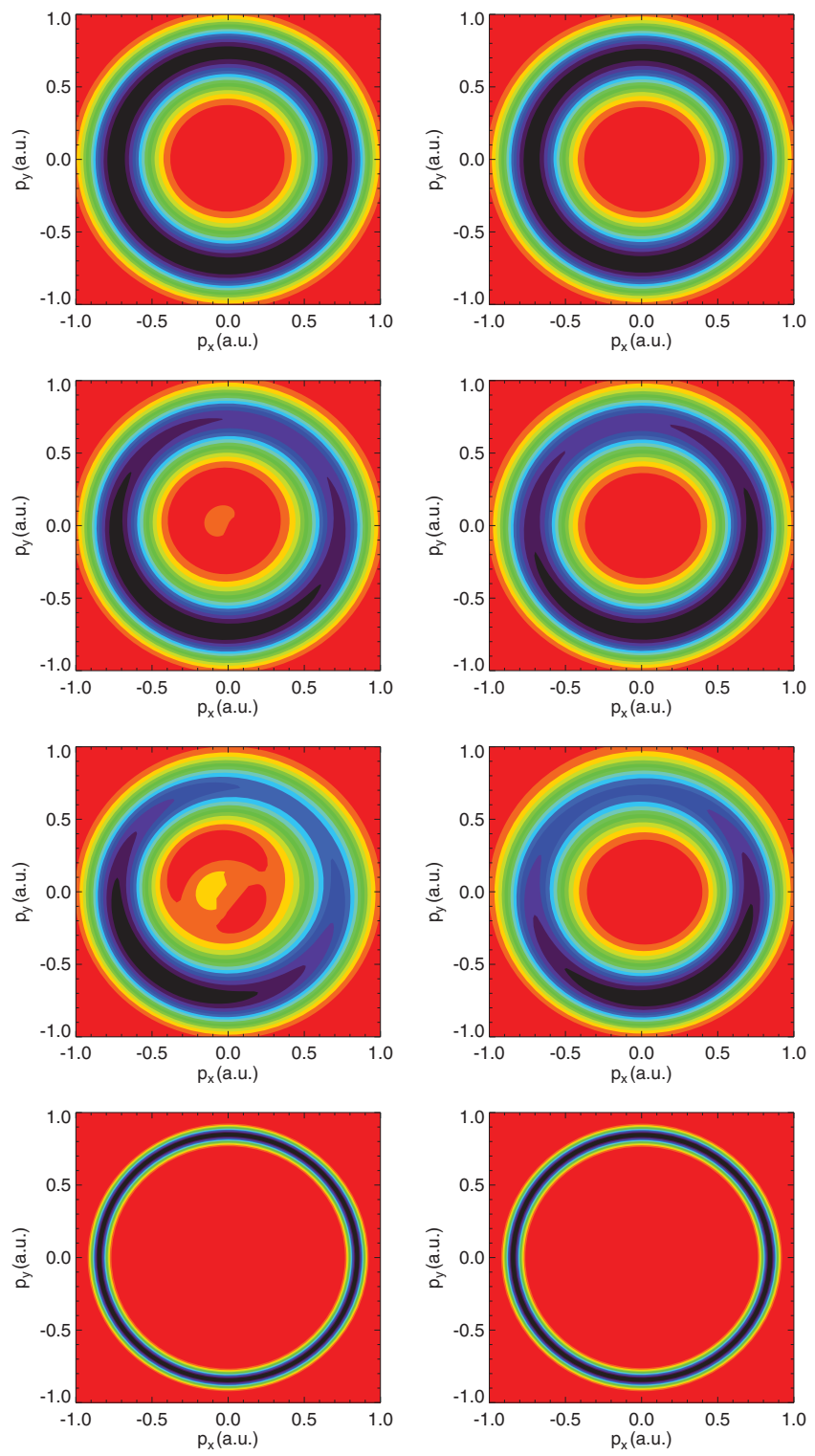

FIG. 2. (Color online) The photoelectron momentum distributions in the equatorial $(x, y)$ plane for Li ionization from the $2 p_{+1}$ state (left column) and $2 p_{-1}$ state (right column). The field parameters are $\omega=13.6 \mathrm{eV}$, the pulse durations are 3 optical cycles (upper three rows) and 10 optical cycles (bottom row), and $\mathcal{E}=0.01$ a.u. (first row), $\mathcal{E}=0.05$ a.u. (second row), and $\mathcal{E}=0.1$ a.u. (third and fourth rows).

noticeable angular anisotropy. The angular dependence of the time delay has a character of a $\pi$-periodic modulation. This effect depends on the pulse duration and disappears completely for a longer pulse of $T_{1} / T=10$. Another observation is that for all the pulse lengths and pulse strengths, the time delay for the corotating electron $2 p_{+1}$ is larger than the time delay for the electron ionized from the $2 p_{-1}$ state.

The origin of these effects can be elucidated most readily within the LOPT. The corresponding expression for the ionization amplitude $a_{k}$ reads

$$
a_{\boldsymbol{k}}=-i \int_{-T_{1} / 2}^{T_{1} / 2}\left\langle\psi_{\boldsymbol{k}}^{(-)}\left|\hat{H}_{\mathrm{int}}(\tau)\right| \psi_{i}\right\rangle e^{i\left(E_{k}-E_{0}\right) \tau} d \tau,
$$


FIG. 3. (Color online) The photoelectron time delays as functions of the polar angle $\phi$ in the equatorial plane for the field strengths $\mathcal{E}=0.01$ a.u. (top), $\mathcal{E}=0.05$ a.u. (middle), and $\mathcal{E}=0.1$ a.u. (bottom). Pulse duration: $T_{1} / T=3$ (left), $T_{1} / T=10$ (right). Solid line (red online), ionization from the $2 p_{+1}$ state; dashed line (green online), ionization from $2 p_{-1}$ state. Short dashed line (blue online), $\tau_{0}^{d}=d \delta_{2} / d E ;$ dots (magenta online), $\tau_{0}^{s}=d \delta_{0} / d E$.

where $\psi_{i}$ is the initial electron state. By plugging expressions (3) into the electromagnetic interaction operator $\hat{H}_{\text {int }}(t)$ in the length gauge, and writing the scalar product $\boldsymbol{E} \cdot \boldsymbol{r} \propto E_{x} x+$ $E_{y} y=-E_{1} r_{-1}-E_{-1} r_{1}$ in the spherical coordinates [19], we obtain

$$
\hat{H}_{\mathrm{int}}(t)=\frac{\mathcal{E}}{\sqrt{2}} f(t)\left(e^{i \omega t} r_{-1}-e^{-i \omega t} r_{1}\right) .
$$

By using the partial wave expansion (5) for the scattering state $\psi_{k}^{(-)}$and setting the initial electron state to $2 p_{ \pm 1}$ we transform the perturbative expression (9) into

$$
\begin{aligned}
a_{k}= & \sum_{l=0,2} \sum_{\mu=-l}^{l} i^{-l} e^{i \delta_{l}} Y_{l \mu}\left(\boldsymbol{n}_{\boldsymbol{k}}\right)\langle k l\|r\| 2 p\rangle \\
& \times\left[A\left(\begin{array}{ccc}
l & 1 & 1 \\
-\mu & -1 & m
\end{array}\right)-B\left(\begin{array}{ccc}
l & 1 & 1 \\
-\mu & 1 & m
\end{array}\right)\right],
\end{aligned}
$$

where

$$
\begin{aligned}
& A=\frac{\mathcal{E}}{\sqrt{2}} \int_{-T_{1} / 2}^{T_{1} / 2} f(t) e^{i\left(E_{k}+\omega-E_{0}\right) t} d t, \\
& B=\frac{\mathcal{E}}{\sqrt{2}} \int_{-T_{1} / 2}^{T_{1} / 2} f(t) e^{i\left(E_{k}-\omega-E_{0}\right) t} d t,
\end{aligned}
$$


and the reduced matrix element $\left\langle k l\|r\| n_{i} l_{i}\right\rangle$ is given by

$$
\left\langle k l|r| n_{i} l_{i}\right\rangle=\hat{l} \hat{l}_{i}\left(\begin{array}{llr}
l & 1 & l_{i} \\
0 & 0 & 0
\end{array}\right) \int r^{2} d r R_{k l}(r) r R_{n_{i} l_{i}}(r),
$$

with $\hat{l}=\sqrt{2 l+1}$. The terms with the coefficients $A$ and $B$ in the square brackets on the right-hand side of Eq. (12) describe emission and absorption of a photon, respectively.

It is easy to see that $A=0$ on the energy shell where $E_{q}=$ $q^{2} / 2=\omega+E_{0}$. On the energy shell Eq. (12) can be rewritten, therefore, as

$$
a_{\boldsymbol{q}}=\left\{\begin{array}{ll}
\alpha_{0,0,-1} Y_{00}\left(\boldsymbol{n}_{\boldsymbol{q}}\right)+\alpha_{2,0,-1} Y_{20}\left(\boldsymbol{n}_{\boldsymbol{q}}\right), & m=-1 \\
\alpha_{2,2,1} Y_{22}\left(\boldsymbol{n}_{\boldsymbol{q}}\right), & m=+1
\end{array},\right.
$$

where

$$
\alpha_{l, \mu, m}=-B i^{-l} e^{i \delta_{l}}\langle q l\|r\| 2 p\rangle\left(\begin{array}{ccc}
l & 1 & 1 \\
-\mu & 1 & m
\end{array}\right) .
$$

Dependence of the amplitudes on the direction of the photoelectron escape is contained in the spherical harmonics appearing in Eq. (13). If we consider the modulus squared of the amplitude in the Eq. (13) in the equatorial plane, we obtain the probability independent of the polar angle, a LOPT prediction which the TDSE calculation for weak fields illustrated in Fig. 2 conforms to.

The situation is different for the time-delay calculations. Indeed, according to Eq. (7), to compute the time delay we have to calculate energy derivative of the amplitude. This includes the energy derivative of the coefficients $A$ and $B$ in Eq. (12). It is easy to see that on the energy shell $d B / d E=0$, while $d A / d E \neq 0$. Moreover, $d A / d E$ depends rather sensitively on the pulse duration as shown in Fig. 4.

For the energy derivative of the ionization amplitude from the $2 p_{-1}$ state we then obtain

$$
\frac{d a_{\boldsymbol{q}}}{d E}=\frac{d \alpha_{0,0,-1}}{d E} Y_{00}\left(\boldsymbol{n}_{\boldsymbol{q}}\right)+\frac{d \alpha_{2,0,-1}}{d E} Y_{20}\left(\boldsymbol{n}_{\boldsymbol{q}}\right)+\eta Y_{2-2}\left(\boldsymbol{n}_{\boldsymbol{q}}\right),
$$

where the coefficient $\eta$ is proportional to the derivative $d A / d E$ computed on the energy shell. In the equatorial plane, the presence of the term with $Y_{2-2}(\boldsymbol{q})$ introduces a modulation proportional to $\exp (-2 i \phi)$. This $\pi$-periodic modulation will also be present in the time delay (7), which is a logarithmic

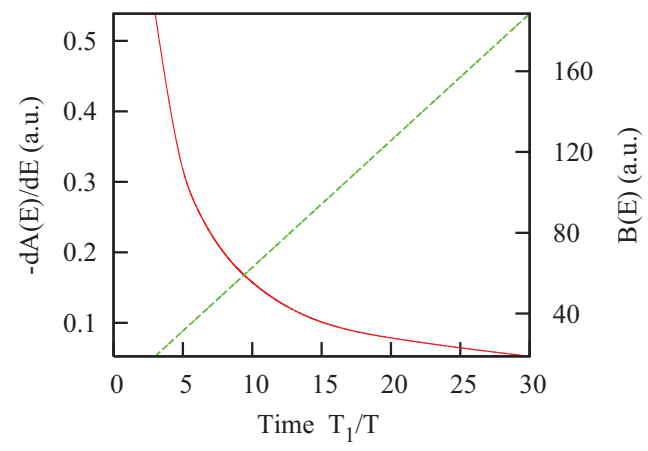

FIG. 4. (Color online) Coefficients $-d A / d E$ (solid line, red online) and $B(E)$ (dashed line, green online) on the energy shell as functions of the pulse duration $T_{1} / T$. The envelope function $f(t)$ is defined in Eq. (3) derivative of the amplitude. The depth of the modulation is determined by the value of the derivative $d A / d E$ on the energy shell, which, in turn, is determined by the particular pulse shape and pulse duration. As we observe in Fig. 4, the derivative $d A / d E$ decreases quickly with the pulse duration. Consequently, the depth of the modulation decreases with increasing pulse length.

No modulation effect is present in the $2 p_{+1}$ case under the perturbative regime. Emission of a photon in this case leads to a continuum state with $l=\mu=2$, which does not introduce angular dependence for the time delay. These LOPT effects are clearly seen in our TDSE calculation in the weak field regime, which are illustrated on the top row of panels in Fig. 3. The $\pi$ modulation of the time delay for the $2 p_{-1}$ is clearly visible for the 3-cycle laser pulse and disappears completely for the 10 -cycle pulse.

As for the relative values of the time delays for ionization from $2 p_{ \pm 1}$ states, this question can be easily addressed using the LOPT expressions. Formulas are simpler for the case of a long pulse, when the term with the coefficient $\eta$ is negligible in Eq. (15). In this case the time delay for the ionization from the $2 p_{+1}$ state is simply $\tau_{0}\left(2 p_{+1}\right)=d \delta_{2} / d E$. The time delay ratio between the $2 p_{-1}$ and $2 p_{+1}$ states can be expressed as

$$
\tau_{0}\left(2 p_{-1}\right) / \tau_{0}\left(2 p_{+1}\right) \approx \frac{(1+\gamma \cos \Delta)}{(1+\gamma \cos \Delta)^{2}+\sin ^{2} \Delta}<1,
$$

where $\gamma=\alpha_{0,0,-1} Y_{00}\left(\boldsymbol{n}_{\boldsymbol{q}}\right)\left[\alpha_{2,0,-1} Y_{20}\left(\boldsymbol{n}_{\boldsymbol{q}}\right)\right]^{-1}$ and $\Delta=\delta_{0}-$ $\delta_{2}$. Here we used the fact that for the presently considered photoelectron energies $d \delta_{2} / d E \gg d \delta_{0} / d E$.

This property of the scattering phase shifts in the field of the $\mathrm{Li}^{+}$ion is illustrated in Fig. 5. At lower photoelectron energies, the scattering phases both in the $s$ wave $(l=0)$ and the $d$ wave $(l=2)$ display the Coulomb singularity. At larger photoelectron energy, $\delta_{0}$ acquires a constant shift due to an occupied $1 s^{2}$ orbital of the matching angular momentum in the ionic core (the Levinson-Seaton theorem [20]). No such orbital exists to match the $d$ wave and hence $\delta_{2}$ tends to zero at large photon energies. This turnover from the growing to falling energy dependence of the $s$ phase at the photon energy of about $15 \mathrm{eV}$ makes the energy derivative $d \delta_{0} / d E$ small while the constantly growing $d$ phase keeps $d \delta_{2} / d E$ large in this photon energy range.

To explain the features of the delays for higher field strength, we have to go beyond the LOPT. As we have already observed in Fig. 1, two-photon ionization processes are clearly contributing, but their role is not very important in forming the photoelectron spectrum even for fields as high as $\mathcal{E}=0.1$ a.u. The situation is rather different for the time delays. We see in Fig. 3 that for the short pulse duration and a large field intensity the time delays are modulated but, unlike in the case of the weak field, the period of modulation is $2 \pi$.

A simple numerical test allows us to clarify the origin of this modulation. We note that absorption and emission of a single photon from the circularly polarized field changes the electron angular momentum projection by \pm 1 , so we can gauge the importance of different ionization channels just by evaluating contributions of different $\mu$ states in the expansion (4). Results of such reduced calculations are shown in Fig. 6, where we suppressed the contributions of the terms with $\mu=1,3$ in 

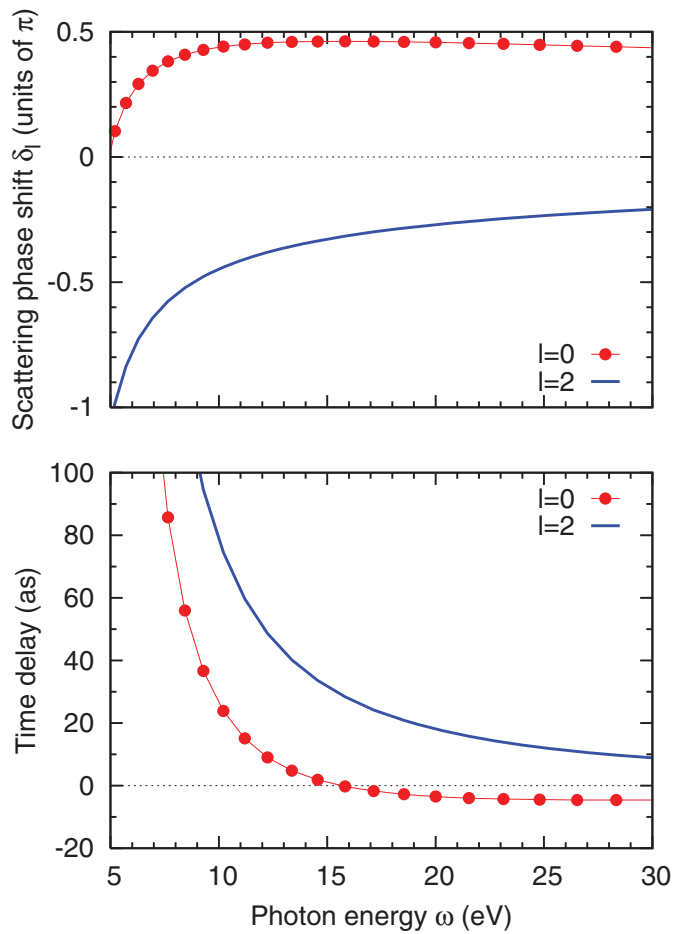

FIG. 5. (Color online) Top: scattering phase shifts $\delta_{l=0}$ (dotted line, red online) and $\delta_{l=2}$ (solid line, blue online) ionization channels. Bottom: the energy derivatives $d \delta_{l=0} / d E$ (dotted line, red online) and $d \delta_{l=2} / d E$ (solid line, blue online) are converted to the units of time delay.

Eq. (4) for ionization from the $2 p_{+1}$ state and terms with $\mu= \pm 1$ for ionization from the $2 p_{-1}$ state. This excludes the second-order processes with participation of two photons and removes the modulation of the time delay completely. For the $2 p_{+1}$ initial state, the final state with $\mu=1$ arises as a result of emission and absorption of a photon, while to get to the $\mu=3$ final state the atom has to absorb two photons from



FIG. 6. (Color online) The time delays as functions of the polar angle $\phi$ in the equatorial plane for the field strength $\mathcal{E}=0.05$ a.u. and the pulse duration $T_{1} / T=3$. The full TDSE calculations for ionization from the $2 p_{+1}$ and $2 p_{-1}$ states are plotted with the solid (red online) and dashed (green online) lines, respectively. The reduced TDSE calculations with the $\mu=1,3$ terms suppressed in Eq. (4) for the $2 p_{+1}$ state and $\mu= \pm 1$ terms suppressed for the $2 p_{-1}$ state are drawn with the short dashed (blue online) and dotted (purple online) lines, respectively. the circularly polarized field. A similar observation applies to the initial state with $2 p_{-1}$. Both these second-order processes lead to the electron states which are far in energy from the energy shell. Their contribution, therefore, should decay very rapidly with the increase of the pulse duration. The plots of Fig. 3 support this conclusion: $2 \pi$-periodic modulation effect is clearly visible for $\mathcal{E}=0.05$ and 0.1 a.u. for the laser pulse duration of $T_{1} / T=3$ and disappears completely for a longer pulse with $T_{1} / T=10$.

\section{CONCLUSION}

We performed time-delay calculations for the photoionization process driven by a circularly polarized laser pulse. We considered initial electron states that are co- and counterrotating with respect to the electric field vector. We found that, similarly to the photoelectron spectra studied extensively in the literature, the time delays are markedly different for these two orientations, depending sensitively on the field strengths and pulse durations. The time delays for the short pulses exhibit modulation effect with respect to the polar angle in the equatorial plane. The period of this modulation varies with the strength of the electric field. For stronger fields the modulation patterns of the time delays for co- and counter-rotating electrons look similar: Both patterns can de described as $2 \pi$ modulation superimposed on a nearly flat background. This effect can be explained as manifestation of the two-photon processes which do not conserve energy and contribute noticeably only in the case of the ionization by a short laser pulse. The situation is different in the weak-field limit, where modulation effect is present only for the time delay of a counter-rotating electron and can be described as a $\pi$ periodic modulation on a constant background. Perturbation theory analysis allowed us to identify the precise absorption mechanisms responsible for this effect.

We employed in our calculations electric fields described by the expressions (3) with the cosine squared envelope function $f(t)$. Another representation of the electric field of a laser pulse frequently used [15] is the one using the cosine squared envelope function for the vector potential describing the field. For short laser pulses, where envelope function can vary quickly, the fields given by these two representations for the same peak field peak strength can have very different temporal profiles. We would have obtained, nevertheless, qualitatively similar conclusions had we represented the fields using the cosine squared envelope function for the vector potential instead of electric field. This can be most easily seen if we write perturbative equations (12) using the velocity form of the field-atom interaction Hamiltonian. The coefficients $A$ and $B$ in the velocity gauge analog of the perturbative expression (12) would have then the same form, leading thus to the similar conclusions.

\section{ACKNOWLEDGMENT}

The authors acknowledge support of the Australian Research Council in the form of the Discovery Grant DP120101805. Resources of the National Computational Infrastructure (NCI) facility were employed. 


\section{APPENDIX}

To describe electron motion with a given asymptotic momentum $\boldsymbol{q}$ we project the solution of the TDSE after the end of the pulse on the set (5) of the scattering states $\psi_{k}^{(-)}(\boldsymbol{r})$ with momenta $\boldsymbol{k}$ centered about the asymptotic momentum $\boldsymbol{q}$. The wave packet describing electron motion can therefore be represented as

$$
\tilde{\Psi}(t)=\int_{\Omega} a_{k} \phi_{k}^{-} e^{-i E_{k} t} d \boldsymbol{k}
$$

where the coefficients $a_{k}$ defined in (6) do not depend on time for the field-free motion of the electron after the end of the laser pulse. Integration domain $\Omega$ in this equation defines the spread of this distribution in momentum space. We can, for example, define integration domain as $|\boldsymbol{k}-\boldsymbol{q}|<\rho$ with some $\rho$.

We are interested in the quantity $r(t)=\boldsymbol{n}_{q} \boldsymbol{r}(t)$, the projection of the trajectory followed by the crest of the electron wave packet on the direction of the unit vector $\boldsymbol{n}_{\boldsymbol{q}}=\boldsymbol{q} / \boldsymbol{q}$. This quantity does not depend on the spread of the electron wave packet; it is determined by the behavior of the amplitude coefficients $a_{\boldsymbol{k}}$ in the immediate vicinity of the point $\boldsymbol{k}=\boldsymbol{q}$.

It is not difficult to obtain asymptotic equation for this function. Following standard prescriptions of the saddle-point method, using large- $r$ asymptotic expressions for the scattering states $\phi_{k}^{-} \propto e^{i \boldsymbol{k} \cdot \boldsymbol{r}+i \gamma(\boldsymbol{r}, \boldsymbol{k})}$ and writing $a_{\boldsymbol{k}}=\left|a_{\boldsymbol{k}}\right| e^{i \delta(\boldsymbol{k})}$ for the wave-packet coefficients, one can easily obtain for large $t$

$$
r(t) \sim q t-\left.\boldsymbol{n}_{q} \frac{d \gamma(\boldsymbol{q} t, \boldsymbol{k})}{d \boldsymbol{k}}\right|_{k=q}-\left.\boldsymbol{n}_{q} \frac{d \delta(\boldsymbol{k})}{d \boldsymbol{k}}\right|_{\boldsymbol{k}=\boldsymbol{q}},
$$

where $\gamma(\boldsymbol{u}, \boldsymbol{v})=\frac{1}{v} \ln (u v+\boldsymbol{u} \boldsymbol{v})$. The term containing the derivative of $\gamma(\boldsymbol{u}, \boldsymbol{v})$ gives the well-known Coulomb corrections to the trajectory. This term grows logarithmically with $t$ for large $t$.

If phase $\delta(\boldsymbol{q})$ depends only on $q$, then we can put in the last equation $\frac{d \delta(\boldsymbol{q})}{d \boldsymbol{q}}=\boldsymbol{q} \frac{d \delta(E)}{d E}$, where $E=q^{2} / 2$. Equation (A2) can then be rewritten as

$$
r(t) \sim q\left(t-\frac{d \delta(E)}{d E}\right)-\left.\boldsymbol{n}_{\boldsymbol{q}} \frac{d \gamma(\boldsymbol{q} t, \boldsymbol{v})}{d \boldsymbol{v}}\right|_{\boldsymbol{v}=\boldsymbol{q}},
$$

where the term with the $d \delta(E) / d E$ is a familiar expression for the Wigner time delay. In the case we consider here, where the phase and hence the time delay do depend on the direction of asymptotic electron momentum $\boldsymbol{q}$, we have to use for the time delay a more general expression following from Eq. (A2):

$$
\tau_{0}=\left.\frac{\boldsymbol{n}_{\boldsymbol{q}}}{q} \frac{d \delta(\boldsymbol{k})}{d \boldsymbol{k}}\right|_{\boldsymbol{k}=\boldsymbol{q}}=\left.\operatorname{Im}\left(\frac{\boldsymbol{n}_{\boldsymbol{q}}}{q} \frac{\partial a_{\boldsymbol{k}}}{\partial \boldsymbol{k}}\right)\right|_{\boldsymbol{k}=\boldsymbol{q}} .
$$

[1] K. Rzazewski and B. Piraux, Phys. Rev. A 47, R1612 (1993).

[2] J. Zakrzewski, D. Delande, J. C. Gay, and K. Rzazewski, Phys. Rev. A 47, R2468 (1993).

[3] I. Barth and O. Smirnova, Phys. Rev. A 84, 063415 (2011).

[4] P. Eckle, A. N. Pfeiffer, C. Cirelli, A. Staudte, R. Dörner, H. G. Muller, M. Büttiker, and U. Keller, Science 322, 1525 (2008).

[5] E. P. Wigner, Phys. Rev. 98, 145 (1955).

[6] C. A. A. de Carvalho and H. M. Nussenzveig, Phys. Rep. 364, 83 (2002).

[7] M. Schultze, M. Fieß, N. Karpowicz, J. Gagnon, M. Korbman, M. Hofstetter, S. Neppl, A. L. Cavalieri, Y. Komninos, T. Mercouris et al., Science 328, 1658 (2010).

[8] S. Nagele, R. Pazourek, J. Feist, K. Doblhoff-Dier, C. Lemell, K. Takasi, and J. Burgdörfer, J. Phys. B 44, 081001 (2011).

[9] D. Shafir, H. Soifer, B. D. Bruner, M. Dagan, Y. Mairesse, S. Patchkovskii, M. Y. Ivanov, O. Smirnova, and N. Dudovich, Nature (London) 485, 343 (2012).

[10] I. A. Ivanov, Phys. Rev. A 86, 023419 (2012).
[11] A. S. Kheifets, I. A. Ivanov, and I. Bray, J. Phys. B 44, 101003 (2011).

[12] M. Schuricke, G. Zhu, J. Steinmann, K. Simeonidis, I. Ivanov, A. Kheifets, A. N. Grum-Grzhimailo, K. Bartschat, A. Dorn, and J. Ullrich, Phys. Rev. A 83, 023413 (2011).

[13] A. Sarsa, F. J. Gálvez, and E. Buendia, At. Data Nucl. Data Tables 88, 163 (2004).

[14] M. Nurhuda and F. H. M. Faisal, Phys. Rev. A 60, 3125 (1999).

[15] A. N. Grum-Grzhimailo, B. Abeln, K. Bartschat, D. Weflen, and T. Urness, Phys. Rev. A 81, 043408 (2010).

[16] I. A. Ivanov, Phys. Rev. A 83, 023421 (2011).

[17] A. S. Kheifets and I. A. Ivanov, Phys. Rev. Lett. 105, 233002 (2010).

[18] I. A. Ivanov and A. S. Kheifets, Phys. Rev. A 85, 021401 (2012).

[19] D. A. Varshalovich, A. N. Moskalev, and V. K. Khersonskii, Quantum Theory of Angular Momentum (World Scientific, Singapore, 1988).

[20] L. Rosenberg, Phys. Rev. A 52, 3824 (1995). 